IOS Press

\title{
Susceptibility-Weighted Imaging in Dementia with Lewy Bodies
}

\author{
Giovanni Rizzo $^{\mathrm{a}, \mathrm{b}, 1, *}$, Roberto De Blasi ${ }^{\mathrm{c}, 1}$, Rosa Capozzo ${ }^{\mathrm{d}, \mathrm{e}}$, Rosanna Tortelli,e \\ Maria Rosaria Barulli ${ }^{\mathrm{d}, \mathrm{e}}$, Rocco Liguori ${ }^{\mathrm{a}, \mathrm{b}}$, Daniela Grasso ${ }^{\mathrm{f}}$ and Giancarlo Logroscino ${ }^{\mathrm{d}, \mathrm{e}}$ \\ ${ }^{a}$ IRCCS Istituto delle Scienze Neurologiche di Bologna, Bologna, Italy \\ ${ }^{\mathrm{b}}$ Department of Biomedical and Neuromotor Sciences, Neurology Unit, University of Bologna, Bologna, Italy \\ ${ }^{\mathrm{c}}$ Department of Diagnostic Imaging, Pia Fondazione di Culto e Religione "Card. G. Panico", Tricase, Italy \\ ${ }^{\mathrm{d}}$ Department of Clinical Research in Neurology, University of Bari, Pia Fondazione di Culto e Religione \\ "Card. G. Panico", Tricase, Italy \\ ${ }^{\mathrm{e}}$ Department of Basic Medical Science, Neuroscience and Sense Organs, University of Bari, Bari, Italy \\ ${ }^{\mathrm{f}}$ IRCCS Casa Sollievo della Sofferenza, San Giovanni Rotondo, Italy
}

Handling Associate Editor: Marco Bozzali

Accepted 23 October 2018

\begin{abstract}
We assessed nigral dorsolateral hyperintensity (swallow tail sign) at susceptibility-weighted imaging using 3T-MRI in 15 dementia with Lewy bodies (DLB), 11 Alzheimer's disease (AD), and 8 frontotemporal dementia (FTD) patients and 10 subjects with subjective memory complaint (SMC). More DLB patients lacked nigral hyperintesity $(p<0.05)$. Sensitivity, specificity, and accuracy of DLB diagnosis were, respectively: $80 \%, 64 \%$, and $73 \%$ versus AD; $80 \%, 75 \%$, and $78 \%$ versus FTD; and $80 \%, 90 \%$, and $84 \%$ versus SMC. Considering bilateral loss, sensitivity decreased (53\%) but specificity increased $(82-100 \%)$. Swallow tail sign loss, especially if bilateral, can be useful for DLB diagnosis.
\end{abstract}

Keywords: Dementia with Lewy bodies, magnetic resonance imaging, nigrosome, swallow tail sign, susceptibility-weighted imaging

\section{INTRODUCTION}

The diagnosis of dementia with Lewy bodies (DLB) may be challenging. Alzheimer's disease (AD) is the most frequent misdiagnosis [1]. The

\footnotetext{
${ }^{1}$ These authors contributed equally to this work.

*Correspondence to: Giovanni Rizzo, Department of Biomedical and Neuromotor Sciences, University of Bologna, and IRCCS Institute of Neurological Sciences of Bologna, Bologna, Italy. Tel.: +39 0514966112; E-mail: g.rizzo@unibo.it.
}

availability of accurate diagnostic tools is crucial. The newly revised DLB diagnostic criteria include the use of imaging biomarkers, e.g., reduced dopamine uptake in basal ganglia demonstrated by SPECT/PET or abnormal ${ }^{123}$ iodine-MIBG myocardial scintigraphy [2]. As for magnetic resonance imaging (MRI), susceptibility-weighted imaging (SWI) is a technique with increasing clinical applications. It allows enhancing the contrast of substances with different magnetic susceptibility to the surrounding tissue background (e.g., iron, calcium). SWI using high field 
MRI scanners (at least 3 Tesla) can detect a dorsolateral hyperintense signal area (also called "swallow tail" sign) in the substantia nigra ( $\mathrm{SN}$ ) of healthy controls [3]. It corresponds to the nigrosome-1, the largest among five clusters of dopaminergic cells in the SN pars compacta, where the maximal dopaminergic neuron loss (90\%) occurs in Parkinson's disease (PD) patients [4]. Recent studies have demonstrated that dorsolateral hyperintense signal area lacks in PD patients, and may distinguish them from healthy controls with high sensitivity and specificity $[5,6]$. Most DLB patients have SN degeneration as in PD. The aim of the present study was to investigate the utility of SWI for detection of the swallow tail sign to differentiate DLB from other degenerative dementias.

\section{METHODS}

We consecutively recruited 15 DLB patients, 12 AD patients, 10 frontotemporal dementia (FTD) patients, and 10 subjects with subjective memory complaint (SMC) at the Department of Clinical Research in Neurology, Pia Fondazione di Culto e Religione "Card. G. Panico", Tricase, Italy from September 2015 to August 2016. Patients fulfilled the appropriate diagnostic criteria [7-9]. All subjects were studied in a 3 Tesla system (Ingenia $^{\circledR}$, Philips Medical System) and a 32channel head coil. Acquisition protocol included axial SWI sequences $(\mathrm{TR}=31, \mathrm{TE}=7.2$, Flip angle $=17$, Voxel size $=0.80 \times 0.81 \times 1.60$, Scan duration $=3 \mathrm{~min}$ ). SMC subjects had no MRI abnormalities. SN was cranio-caudally sampled obtaining five axial sections and displayed by MPR reconstruction starting from an oblique plane, parallel to the corpus callosum. Two neuroradiologists (DG and RDB), blinded to the clinical conditions and to each other's decisions, examined SWI images to classify the subjects according to the presence or loss of the dorsolateral nigral hyperintensity. A third rater (GR) resolved the discordances.

Radiological DLB diagnosis requested the uni- or bilateral loss of nigral hyperintensity.

We followed up all the subjects for at least one year to confirm diagnosis.

Statistical analyses were performed using SPSS 24.0. The differences in demographic and clinical data were evaluated by $\chi^{2}$ test for categorical variables and Kruskal-Wallis test followed by MannWhitney U test for continuous variables. Agreement was tested using Cohen's k statistics. The distribution of the nigral hyperintensity among the groups was tested using $\chi^{2}$ test. As the groups slightly differed in the mean age, we also used logistic regression models with the age at the MRI as covariate. $p<0.05$ was considered significant. The ability to identify DLB was assessed using the clinical diagnosis as gold standard. Sensitivity, specificity, and accuracy were calculated.

The procedures used followed the Helsinki Declaration regarding international clinical research involving human beings. The local Human Ethics Committee approved the study and all subjects gave their written informed consent to the study.

\section{RESULTS}

Two scans (one AD and one FTD) were excluded due to motion artifacts. One FTD patient interrupted MRI scan before SWI acquisition for claustrophobia. Finally, we studied 15 DLB patients (all DLB probable), 11 AD patients (all AD probable), 8 FTD patients (seven behavior variants and one primary progressive aphasia), and 10 SMC subjects. Age slightly differed among the groups, while sex did not. The patients did not differ in disease duration and MMSE scores (Table 1). Raters agreement was $86 \%$ (kappa $=0.71, p<0.0001)$. Twelve out of 15 DLB patients lacked nigral hyperintesity unilaterally or bilaterally, unlike the other groups (AD: 4/11; FTD: 2/8; SMC: $1 / 10 ; p<0.05$. Table 1, Fig. 1). After the correction for the age of the subjects, all the differences remained significant except that between DLB and FTD patients (Table 1). Sensitivity, specificity, and accuracy values are reported in Table 1 .

After one-year follow-up, all diagnoses were confirmed. However, one AD patient, who had only mild parkinsonian symptoms at the time of MRI scan, reported a worsening of the parkinsonism and the appearance of visual hallucinations after a major surgery (7 years after dementia onset). Due to the late occurrence of these symptoms, we confirmed the diagnosis of $\mathrm{AD}$, but considering a mixed type, with concomitant Lewy body (LB) disease [8]. Accordingly, if we use as gold standard the possible presence of LB pathology, including this patient in the LB group, the sensitivity, specificity, and accuracy of SWI increase, respectively, to $81 \%, 70 \%$, and $77 \%$ versus $\mathrm{AD} ; 81 \%, 75 \%$, and $79 \%$ versus $\mathrm{FTD}$; and $81 \%, 90 \%$, and $85 \%$ versus SMC (considering only the bilateral loss: $56 \%, 90 \%$, and $69 \%$ versus $\mathrm{AD}$; $56 \%, 88 \%$, and $67 \%$ versus FTD; and 56\%, $100 \%$, and $73 \%$ versus SMC). 
Table 1

Demographic, clinical and MRI characteristics of subjects at the time of the scan

\begin{tabular}{|c|c|c|c|c|c|}
\hline & $\operatorname{DLB}(n=15)$ & $\mathrm{AD}(n=11)$ & FTD $(n=8)$ & $\operatorname{SMC}(n=10)$ & $p$ \\
\hline $\operatorname{Sex}(M / F)$ & $8 / 7$ & $4 / 6$ & $3 / 5$ & $5 / 5$ & $\mathrm{NS}^{\mathrm{A}}$ \\
\hline Age & $76 \pm 7$ & $74 \pm 8$ & $64 \pm 12$ & $67 \pm 9$ & $<0.05^{\mathrm{B} 1}$ \\
\hline Age at onset & $71 \pm 7$ & $69 \pm 8$ & $61 \pm 13$ & I & $\mathrm{NS}^{\mathrm{B}}$ \\
\hline Disease duration & $5 \pm 3$ & $5 \pm 3$ & $3 \pm 2$ & l & $\mathrm{NS}^{\mathrm{B}}$ \\
\hline MMSE & $17 \pm 6$ & $13 \pm 5$ & $19 \pm 6$ & $28 \pm 2$ & $<0.001^{\mathrm{B} 2}$ \\
\hline Parkinsonism (UPDRS part III) & $15 / 15(27 \pm 8)$ & $1 / 11(13)$ & $1 / 8(15)$ & l & / \\
\hline Visual hallucinations & $15 / 15$ & $0 / 11$ & $0 / 8$ & / & / \\
\hline Fluctuating cognition* & $14 / 15$ & $0 / 11$ & $0 / 8$ & l & / \\
\hline REM sleep behavior disorder ${ }^{\#}$ & $11 / 15$ & $0 / 11$ & $0 / 8$ & l & I \\
\hline Severe neuroleptic sensitivity** & 0/15 (NA:6) & 0/11 (NA:7) & 0/8 (NA:4) & I & I \\
\hline \multicolumn{6}{|c|}{ Loss of dorsolateral nigral hyperintensity } \\
\hline Unilateral+bilateral & $12 / 15(80 \%)$ & $4 / 11(36 \%)$ & $2 / 8(25 \%)$ & $1 / 10(10 \%)$ & $<0.05^{\mathrm{C} 3}$ \\
\hline Unilateral & $4 / 15(27 \%)$ & $2 / 11(18 \%)$ & $1 / 8(12.5 \%)$ & $1 / 10(10 \%)$ & \\
\hline Bilateral & $8 / 15(53 \%)$ & $2 / 11(18 \%)$ & $1 / 8(12.5 \%)$ & $0 / 10(0 \%)$ & \\
\hline
\end{tabular}

Diagnostic accuracy values for the loss of the dorsolateral nigral hyperintensity, using DLB clinical diagnosis as the gold standard Uni- or bilateral loss DLB versus AD DLB versus FTD DLB versus SMC DLB versus non-DLB

Sensitivity

Specificity

Accuracy

Bilateral loss

Sensitivity

Specificity

$80 \%$
$64 \%$
$73 \%$
DLB versus AD
$53 \%$
$82 \%$
$65 \%$

DLB versus FTD
$80 \%$
$75 \%$
$78 \%$
DLB versus FTD
$53 \%$
$88 \%$
$65 \%$

$\begin{array}{cc}\text { DLB versus SMC } & \text { DLB versus non-DLB } \\ 80 \% & 80 \% \\ 90 \% & 76 \% \\ 84 \% & 77 \% \\ \text { DLB versus SMC } & \text { DLB versus non-DLB } \\ 53 \% & 53 \% \\ 100 \% & 90 \% \\ 72 \% & 77 \%\end{array}$

PET and SPECT data were available only for few patients and are not reported in details. DLB, dementia with Lewy bodies; AD, Alzheimer's dementia; FTD, frontotemporal dementia; SMC, subjective memory complaint; MMSE, Mini-Mental State Examination; UPDRS, Unified Parkinson's Disease Rating Scale; NS, not significant. *anamnestic. * anamnestic, no polysomnography. ${ }^{* *}$ NA, not applicable because never treated with neuroleptics. ${ }^{\mathrm{A}}$ Chi-square Test; ${ }^{\mathrm{B}}$ Kruskal-Wallis Test. $1=$ post-hoc Mann-Whitney U test: DLB versus FTD $p<0.05$, DLB versus $\mathrm{SMC} p<0.05 .2=$ post - hoc Mann-Whitney $\mathrm{U}$ test: $\mathrm{DLB}$ versus $\mathrm{SMC} p<0.001$, AD versus $\mathrm{SMC} p<0.001, \mathrm{FTD}$ versus $\mathrm{SMC} p<0.01$. ${ }^{\mathrm{C}}$ Chi-square Test $p<0.001$; post-hoc: DLB versus $\mathrm{AD} p<0.05$, DLB versus FTD $p=0.01$, DLB versus SMC $p=0.001$, DLB versus non-DLB $p<0.001$. Logistic regression using age as covariate $p<0.05$; post-hoc: DLB versus AD $p<0.05$, DLB versus FTD NS, DLB versus SMC $p=0.005$, DLB versus non-DLB $p=0.004$.
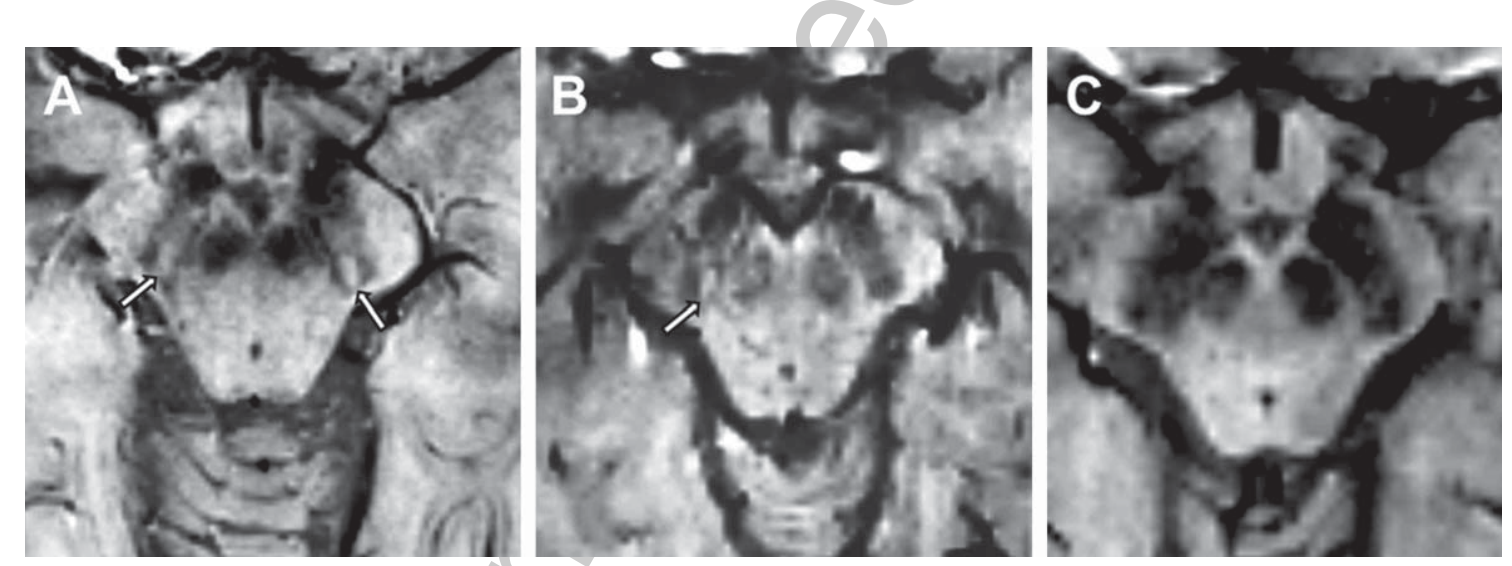

Fig. 1. Examples of normal bilateral dorsolateral nigral hyperintensity (A: AD patient), unilateral loss (B: DLB patient) and bilateral loss (C: DLB patient). The arrows indicate the visible dorsolateral nigral hyperintensities.

from the patients with AD and FTD and SMC. In the pairwise analyses, DLB patients significantly differed from other groups except for FTD, although the prevalence of the swallow tail sign loss was clearly higher in the DLB patients compared with hyperintensity using 3T SWI, disclosing a loss of this sign in the majority of the DLB patients, differently 
FTD patients. The lack of significance was probably due to the smaller number and age of patients with FTD lowering statistical power. However, we cannot exclude that the discrimination between DLB and FTD patients by the swallow tail sign may actually not be optimal. Future studies should better evaluate this point.

In our study, the assessment of dorsolateral nigral hyperintensity was able to identify DLB with good diagnostic accuracy, with a sensitivity of $80 \%$ and a specificity ranging from $64 \%$ (versus $\mathrm{AD}$ ) to $90 \%$ (versus SMC). If we considered only the bilateral loss, sensitivity decreased to $53 \%$ but specificity increased, ranging from $82 \%$ (versus $\mathrm{AD}$ ) to $100 \%$ (versus SMC). Currently, there are only few data on the evaluation of swallow-tail sign in DLB, reported by two recent retrospective studies [10, 11]. A first study on DLB, AD, and mild cognitive impairment subjects performed both a visual evaluation of SWI images reporting as abnormal not only a loss but also a hypointensity of the nigrosome- 1 of $50 \%$ or greater and a semi-quantitative analysis using a contrast ratio between signal of nigrosome- 1 and of midbrain tegmentum [10]. Interestingly, the authors reported a sensitivity and specificity of about $90 \%$ for both approaches, slightly better than DaT-SPECT. However, these approaches, although very accurate, are less simple than identifying an absence or presence of nigral hyperintensity and hence probably more difficult to apply in clinical practice [11]. The second study, which also included some FTD patients, evaluated a heterogeneous sample merging the MRI exams from four different scanners (1.5 or 3 Tesla), probably determining the low agreement (kappa $=0.4)$ and results [11]. They reported an overall sensitivity of $63 \%$, specificity of $79 \%$, and accuracy of $76 \%$, better using 3T SWI and thinner slices. They did not evaluate bilateral loss separately [11]. Our data, using a single 3T scanner and a fixed SWI protocol, show as the evaluation of the swallow tail sign is reliable and has an accuracy to diagnose DLB similar to that reported by the second previous work [11], although the specificity clearly increased in the case of bilateral loss.

Limit of our and previous studies is the lack of pathological diagnosis, as the clinical diagnosis is yet suboptimal [1]. The low sample size and the quite long disease duration of DLB and AD patients should be considered other limitations of this study. Future studies will have to evaluate the swallow tail sign in patients with early-stage disease. Furthermore, they will need to include more patients, especially with
FTD. A minor limit could be the use of SMC subjects as controls. However, the possibility of a subclinical disease was very low. Finally, the slight difference in the age of the subjects could be a further limit, although recent data have suggested that age-related increase in iron concentration does not affect the visibility of swallow tail sign [12]. However, age of DLB and $\mathrm{AD}$ patients did not differ and we performed statistical analyses using the age as covariate.

In conclusion, the assessment of the dorsolateral nigral hyperintensity could be a reliable and noninvasive biomarker of SN degeneration, useful for the clinical diagnosis of DLB, although the accuracy seems to be suboptimal. Remarkably, the bilateral loss increased the diagnostic specificity up to more than $80 \%$ and could be considered a good imaging biomarker of DLB in patients with dementia.

\section{ACKNOWLEDGMENTS}

This research did not receive any specific grant from funding agencies in the public, commercial, or not-for-profit sectors.

Authors' disclosures available online (https:// www.j-alz.com/manuscript-disclosures/18-0687r1).

\section{REFERENCES}

[1] Rizzo G, Arcuti S, Copetti M, Alessandria M, Savica R, Fontana A, Liguori R, Logroscino G (2018) Accuracy of clinical diagnosis of dementia with Lewy bodies: A systematic review and meta-analysis. $J$ Neurol Neurosurg Psychiatry 89, 358-366.

[2] McKeith IG, Boeve BF, Dickson DW, Halliday G, Taylor JP, Weintraub D, Aarsland D, Galvin J, Attems J, Ballard CG, Bayston A, Beach TG, Blanc F, Bohnen N, Bonanni L, Bras J, Brundin P, Burn D, Chen-Plotkin A, Duda JE, ElAgnaf O, Feldman H, Ferman TJ, Ffytche D, Fujishiro H, Galasko D, Goldman JG, Gomperts SN, Graff-Radford NR, Honig LS, Iranzo A, Kantarci K, Kaufer D, Kukull W, Lee VMY, Leverenz JB, Lewis S, Lippa C, Lunde A, Masellis M, Masliah E, McLean P, Mollenhauer B, Montine TJ, Moreno E, Mori E, Murray M, O’Brien JT, Orimo S, Postuma RB, Ramaswamy S, Ross OA, Salmon DP, Singleton A, Taylor A, Thomas A, Tiraboschi P, Toledo JB, Trojanowski JQ, Tsuang D, Walker Z, Yamada M, Kosaka K (2017) Diagnosis and management of dementia with Lewy bodies: Fourth consensus report of the DLB Consortium. Neurology 89, 88-100.

[3] Schwarz ST, Afzal M, Morgan PS, Bajaj N, Gowland PA, Auer DP (2014) The 'swallow tail' appearance of the healthy nigrosome - a new accurate test of Parkinson's disease: A case-control and retrospective cross-sectional MRI study at 3T. PLoS One 9, e93814.

[4] Damier P, Hirsch EC, Agid Y, Graybiel AM (1999) The substantia nigra of the human brain. I. Nigrosomes and 
the nigral matrix, a compartmental organization based on calbindin D(28K) immunohistochemistry. Brain 122, 14211436.

[5] Rizzo G, Zanigni S, De Blasi R, Grasso D, Martino D, Savica $R$, Logroscino G (2016) Brain MR contribution to the differential diagnosis of Parkinsonian syndromes: An update. Parkinsons Dis 2016, 2983638.

[6] Mahlknecht P, Krismer F, Poewe W, Seppi K (2017) Metaanalysis of dorsolateral nigral hyperintensity on magnetic resonance imaging as a marker for Parkinson's disease. Mov Disord 32, 619-623.

[7] McKeith IG, Dickson DW, Lowe J, Emre M, O'Brien JT, Feldman H, Cummings J, Duda JE, Lippa C, Perry EK, Aarsland D, Arai H, Ballard CG, Boeve B, Burn DJ, Costa D, Del Ser T, Dubois B, Galasko D, Gauthier S, Goetz CG, Gomez-Tortosa E, Halliday G, Hansen LA, Hardy J, Iwatsubo T, Kalaria RN, Kaufer D, Kenny RA, Korczyn A, Kosaka K, Lee VM, Lees A, Litvan I, Londos E, Lopez OL, Minoshima S, Mizuno Y, Molina JA, Mukaetova-Ladinska EB, Pasquier F, Perry RH, Schulz JB, Trojanowski JQ, Yamada M; Consortium on DLB (2005) Diagnosis and management of dementia with Lewy bodies: Third report of the DLB Consortium. Neurology 65, 1863-1872.

[8] McKhann GM, Knopman DS, Chertkow H, Hyman BT, Jack CR Jr, Kawas CH, Klunk WE, Koroshetz WJ, Manly JJ, Mayeux R, Mohs RC, Morris JC, Rossor MN, Scheltens P, Carrillo MC, Thies B, Weintraub S, Phelps CH
(2011) The diagnosis of dementia due to Alzheimer's disease: Recommendations from the National Institute on Aging-Alzheimer's Association workgroups on diagnostic guidelines for Alzheimer's disease. Alzheimers Dement 7, 263-269.

[9] Neary D, Snowden JS, Gustafson L, Passant U, Stuss D, Black S, Freedman M, Kertesz A, Robert PH, Albert M, Boone K, Miller BL, Cummings J, Benson DF (1998) Frontotemporal lobar degeneration: A consensus on clinical diagnostic criteria. Neurology 51, 1546-1554.

[10] Kamagata K, Nakatsuka T, Sakakibara R, Tsuyusaki Y, Takamura T, Sato K, Suzuki M, Hori M, Kumamaru KK, Inaoka T, Aoki S, Terada H (2017) Diagnostic imaging of dementia with Lewy bodies by susceptibility-weighted imaging of nigrosomes versus striatal dopamine transporter single-photon emission computed tomography: A retrospective observational study. Neuroradiology 59, 89-98.

[11] Shams S, Fällmar D, Schwarz S, Wahlund LO, van Westen D, Hansson O, Larsson EM, Haller S (2017) MRI of the swallow tail sign: A useful marker in the diagnosis of Lewy body dementia? AJNR Am J Neuroradiol 38, 1737-1741.

[12] Gramsch C, Reuter I, Kraff O, Quick HH, Tanislav C, Roessler F, Deuschl C, Forsting M, Schlamann M (2017) Nigrosome 1 visibility at susceptibility weighted 7T MRIA dependable diagnostic marker for Parkinson's disease or merely an inconsistent, age-dependent imaging finding? PLoS One 12, e0185489. 